\title{
Clinical aspects of diabetes in pregnancy
}

\author{
R. E. ROBINSON \\ F.R.C.S., M.R.C.O.G. \\ Cambridgeshire Area Health Authority (Teaching)
}

\begin{abstract}
Summary
The introduction of insulin produced a rapid improvement in the maternal prognosis in pregnancy associated with diabetes mellitus. Within years, maternal mortality fell from $50 \%$ and has become a rarity. Improvement in fetal survival was less dramatic until the work of Pedersen (1952); Peel and Oakley (1950) and White (1949) demonstrated that with determined antenatal control of diabetes the fetal outcome was greatly improved. Further improvement in the care of the fetus of the pregnant diabetic with fetal monitoring, both ante- and intra-partum, together with modern neonatal expertise, has brought the perinatal and neonatal morbidity and mortality for this group of patients close to that of the non-diabetic. An account is given of the author's present day practice in this group of patients.
\end{abstract}

\section{The diagnosis of diabetes in pregnancy}

Two groups of patients are recognized.

(1) Those in whom the disease has been diagnosed before pregnancy and is being treated with insulin, rarely with oral hypoglycaemic agents or diet alone. (2) Those with gestational diabetes, i.e. diabetes diagnosed during pregnancy.

Complicated subclassifications such as those suggested by White (1949) are unnecessary; they do not affect management, although they may be a guide to prognosis. Furthermore, such classifications imply a concept of mild or severe disease. Such a concept is misleading so far as the fetus is concerned; the maternal disorder may be so mild that there is no subjective maternal deterioration yet so severe for the fetus that it may die.

The age group of pregnant diabetics is such that medical complications of diabetes are rare. A major clinical problem is the diagnosis of gestational diabetes. Currently, more infants die in this group of patients than in the women whose disease is diagnosed before pregnancy, largely owing to failure to diagnose gestational diabetes whilst the fetus is still alive.

Diagnosis of gestational diabetes

Ideally a screening test is required for all pregnant women. The most reliable would be a full oral glucose tolerance test (GTT) at 32 weeks but this is usually logistically impossible. Simple tests, whilst detecting some cases, will not detect all. A constant search for markers for gestational diabetes, e.g. Hb $\mathbf{A}_{1 c}$, continues. Usually, a compromise is effected by establishing criteria for screening high risk groups, namely:

(1) glycosuria on routine testing of urine on 2 or more occasions in pregnancy;

(2) family history of diabetes in sibling, parent or grandparent;

(3) obesity, particularly all patients weighing $>90$ kg;

(4) past history of an infant with a birth weight of $4.25 \mathrm{~kg}$ or when the fetal size in the presen pregnancy seems excessive;

(5) previous, especially unexplained, fetal loss;

(6) hydramnios;

(7) a past history of an infant with neonatal hypoglycaemia.

The GTT is performed at 32 weeks unless clinical disease has been seriously suspected previously, but a normal test in early pregnancy should be followed by a repeat at 32 weeks or later; carbohydrate tolerance steadily deteriorates as pregnancy progresses. It is never too late to perform a GTT whilst the fetus is still alive. However, following fetal death or delivery the GTT may return to normal within $48 \mathrm{hr}$ and a normal result in such circumstances is of no value in relation to management of further pregnancies.

\section{Normal values of the GTT}

The accepted British Diabetic Association definition of the normal GTT includes a fasting and 2-hr level of $6.7 \mathrm{mmol} / \mathrm{l}$ and a peak one-hr level of $10 \mathrm{mmol} / \mathrm{l}$. These criteria are probably too lax for the pregnant state and it is suggested that the criteria of Wright, Dixon and Joplin (1968) be used as follows:

$\begin{array}{ll}\text { Fasting } & 5.0 \mathrm{mmol} / 1 \\ 60 \mathrm{~min} & 8.5 \mathrm{mmol} / \mathrm{l} \\ 90 \mathrm{~min} & 8.0 \mathrm{mmol} / 1 \\ 2 \mathrm{hr} & 7.0 \mathrm{mmol} / \mathrm{l}\end{array}$




\section{Rationale of management}

Management is based on (1) the accurate control of maternal diabetes so that blood glucose is kept in the normoglycaemic range, thereby enabling pregnancy to be safely prolonged without increasing the risk of intra-uterine fetal death. At the same time, as term approaches, successful vaginal delivery becomes feasible owing to normal fetal size and the increasing ripeness of the cervix; (2) exemplary antenatal fetal monitoring to prevent unexpected fetal death in utero before labour; (3) avoidance of intrapartum fetal death and birth injury.

Uncontrolled maternal diabetes leads, in the fetus, to infusion hyperglycaemia, in turn leading to fetal $\beta$-cell hypertrophy and hyperinsulinaemia, resulting in the laying down of fat and protein. It may not be possible to support a large fetus with a placenta of finite capacity and hence the concept of insufficiency is invoked as a cause of unexpected (and unexplained) fetal death. This may not be a matter of gaseous exchange capacity, rather a metabolic insufficiency. If the fetus is unduly large, mechanical difficulties may occur at the time of vaginal delivery with damage to both mother and fetus. Neonatally the high fetal insulin levels existing in utero may lead to profound hypoglycaemia. Finally, despite the advances in the understanding of neonatal respiratory physiology and in the management of its disorders, an unacceptable number of infants suffer from neonatal respiratory complications.

\section{Management of the gestational diabetic}

A GTT is performed in suspicious cases. If normal, no further action is required unless the test has been performed in early pregnancy when it should be repeated at 32 weeks or later.

If the GTT is abnormal, a blood sugar series (BSS) is carried out on a normal diet taking capillary blood at 2-hourly intervals from 6 a.m. to 10 p.m. In many cases, the BSS will be normal, i.e. no level $>7 \mathrm{mmol} / \mathrm{l}$. (It should be remembered that a GTT is a stress test and, if abnormal, does not necessarily mean that the blood glucose levels in normal circumstances will be raised.) The BSS should be repeated fortnightly until term when labour is induced. If the initial or subsequent BSS is abnormal, a 150-g carbohydrate diet is instituted and the BSS repeated $48 \mathrm{hr}$ later. If normal, no further action is required except that the BSS is repeated fortnighty and labour induced at 39 weeks. If after institution of the 150-g carbohydrate diet the BSS is not rendered normal or becomes abnormal after a period of normality, then insulin therapy is required.

Whilst frequent periods of in-patient assessment may be necessary, permanent in-patient observation in this group of patients is unnecessary unless insulin is required.
Management of the 'insulin dependent' pregnant diabetic

As soon as possible, the gestation age is confirmed by ultrasound. The current state of control of diabetes is assessed; this may necessitate admission. Alteration of the patient's insulin regime may be necessary; if control is adequate, no change in regime is indicated. There is increasing interest in management of early pregnancy in the diabetic, the suggestion having been made that poor control at this time may lead to abortion and may possibly be related to the recognized higher incidence of fetal abnormality than in normal pregnancy.

Attention to diet may be required and finally unfavourable factors determined, e.g. retinopathy (which rarely is of obstetric importance), nephropathy or hypertension (which are of serious import).

The patient is taught to test her urine for sugar and acetone 4 times daily and to record it. She has the object of management carefully explained; most patients will take a lively interest and great pride in their control. They must know that at any time advice is available from, or they may admit themselves to, the antenatal ward.

The patient is seen at 3-weekly intervals throughout pregnancy, ideally in a joint clinic with a physician and obstetrician, or failing this by an obstetrician with a special interest and training in the care of pregnant diabetics.

Final admission for accurate stabilization is at $\mathbf{3 2}$ weeks but earlier if control has been poor. After 32 weeks, once control is well established, weekend leave is usually allowed. The freedom with which out-patient management is permitted is governed largely by the stability of the patient's control and the distance from her home to the hospital.

\section{The insulin regime}

A twice daily injection of a mixture of soluble and isophane insulin is used. A rough guide to the starting dose at 32 weeks is as follows: Determine the total number of units of insulin administered per day before admission and add $25 \%$. The total dose is divided into 2 , giving half at $6.00 \mathrm{a.m}$. and half at 6.00 p.m. and of each dose one third should be as soluble and two thirds as isophane.

As a working rule, soluble insulin controls the blood glucose for the first $6 \mathrm{hr}$ and isophane 6 to $12 \mathrm{hr}$ after injection. Blood sugar series are initially carried out 3 times weekly and the insulin dosage adjusted until excellent control is obtained, then twice weekly. The aim is to keep the blood glucose between 3.5 and $6 \mathrm{mmol} / \mathrm{l}$. Most patients are used to much more rigid control than this. If their blood glucose levels are brought down too rapidly, then they may experience hypoglycaemic attacks which in turn deters them from co-operation in reducing the 
blood glucose to obstetrically acceptable levels. However, if this is achieved slowly over several days there is rarely any difficulty and the levels described as ideal are then tolerated without symptoms. Intrauterine fetal hypoglycaemia is probably an exaggerated risk; maternal hypoglycaemic symptoms would arise before a level harmful to the fetus is reached. If the maternal blood glucose level is excellent, the fetus will not become hyperinsulinaemic.

Attempts to monitor maternal control by urine testing are useless. Firstly, the levels of blood glucose aimed at are well below the usual renal threshold and, secondly, the renal threshold is not only highly variable from patient to patient but also in the same patient from day to day.

\section{Diet}

Commonly little or token attention to diet has been paid before pregnancy. Rigorous attention is essential during pregnancy. Until 32 weeks, depending on the maternal size and activity, a 150-180-g carbohydrate diet is usually sufficient. However, in 2 circumstances, more carbohydrate may be required; the first, where there is persistent ketonuria but normal blood glucose levels indicating excess fat metabolism due to insufficient carbohydrate intake and, the second, where there is excess glucose loss in the urine due to a low renal threshold. This latter situation may also be accompanied by ketonuria and only blood-sugar estimations will reveal that diabetic keto-acidosis is not impending.

\section{Routine observations whilst in hospital}

\section{Each day}

BP measurement

Urine testing 4 times for glucose, ketone bodies and protein

Abdominal palpation

Fetal heart recording

Fetal movement chart

Cardiotacography (CTG) if a problem with the fetus is suspected

Twice weekly

CTG

Twenty-four hr oestriol excretion

Blood sugar series

Weekly

Maternal weight and girth

Plot of total insulin requirement in $24 \mathrm{hr}$

Ultrasonic measurement of bi-parietal diameter and body girth

At 37 weeks

Ultrasonic localization of placenta and amniocentesis for lecithin/sphingomyelin ratio

Plain abdominal X-ray to exclude fetal abnormality

Decision re mode and time of delivery. Provided all is proceeding satisfactorily, delivery is planned for 38 weeks.
Problems

The patient becomes too difficult to control

This is usually because she is not adhering to her diet or because there is marked fluctuation in her activity. Occasionally a patient may be encountered who is genuinely difficult to control, particularly if there is a large and variable urinary loss of glucose.

The patient is unwilling to remain in hospital

This is fortunately rare; provided frequent attendance is possible as an out-patient, the control achieved may be reasonable but is often indifferent, and early delivery becomes necessary.

\section{Signs of fetal compromise}

Falling maternal weight or maternal abdominal girth; static or falling urinary oestriol excretion; reduced fetal movements; large baby or polyhydramnios; falling insulin requirements.

\section{The choice of method of delivery}

Vaginal delivery is anticipated when control is satisfactory and 38 weeks' gestation achieved. Induction then is more likely to be successful than at a more premature stage and should be increasingly so with newer methods of cervical ripening.

Elective Caesarean section is chosen where there is any complication added to the maternal diabetes namely hypertension, renal complications, malpresentation, previous Caesarean section, advanced maternal age or obesity. In addition, Caesarean section is indicated if control has been difficult or if there is evidence of fetal compromise usually entailing delivery before 38 weeks.

The lecithin/sphingomyelin ratio should be $>2: 1$. In diabetics, this test may be less reliable than in the non-diabetic in identifying an infant which may encounter respiratory difficulty.

Where doubt exists, elective Caesarean section should be chosen. There is frequently too much 'pride' in the achievement of vaginal delivery for diabetics, a condition which in itself is probably more of an indication for Caesarean section than many other conditions for which Caesarean section is currently employed.

\section{Management for elective Caesarean section}

Established diet and insulin dosage is maintained until the day before delivery. On the morning of delivery the patient's usual morning dose of soluble insulin is given, an i.v. infusion of $5 \%$ dextrose is set up and an i.v. bolus of $50 \mathrm{ml}$ of $50 \%$ dextrose is given into the drip tubing. Caesarean section is then performed within the next $3 \mathrm{hr}$ under general or epidural anaesthesia.

\section{Management for vaginal delivery}

Exactly the same procedure is followed as for 
elective Caesarean section. An epidural anaesthetic is inserted. Amniotomy is performed, a fetal scalp electrode applied and continuous fetal monitoring instituted until delivery. Intravenous oxytocin is begun at once. Most patients will deliver within $8 \mathrm{hr}$ but if undelivered at that time, it will be evident whether or not vaginal delivery is likely to occur within the next $4 \mathrm{hr}$ and if not, then attempt at vaginal delivery should be abandoned before maternal metabolic difficulty or fetal distress supervene. During labour, 2-hourly blood glucose should be estimated. With this schedule, it is unusual to find that either hypo- or hyperglycaemia demanding treatment occur.

\section{After delivery}

The i.v. infusion should be continued for $12 \mathrm{hr}$. Insulin is often not required for $24 \mathrm{hr}$. Indeed, in gestational diabetes insulin is often not needed at all after delivery. In patients who were insulin dependent before pregnancy, a sliding scale schedule is required for $48 \mathrm{hr}$ and thereafter a return to the pre-pregnancy regime may be instituted, although with some modification in the event of breast feeding.

\section{The infant}

Whilst a paediatrician should always be present at delivery, with present fetal monitoring techniques, resuscitation should rarely be required. Most infants need no special care (but nevertheless should be delivered where such facilities are available) and may be warded with their mothers. Blood glucose levels should be measured every $2 \mathrm{hr}$. The infant should be fed as soon as reasonably possible and careful observation for respiratory difficulties is essential. With impeccable control of maternal diabetes, fetal problems will be rare, but in less well controlled cases the following problems may occur requiring special and intensive care facilities: hypoglycaemia; respiratory difficulty including respiratory distress syndrome; jaundice; hypocalcaemia. Amongst diabetic mothers, the commonest cause of fetal loss is early and mid-trimester abortion and fetal malformation.

In summary, meticulous attention to the antenatal management of diabetes, antenatal and intra-partum fetal monitoring with excellent neonatal care will produce exemplary results. However, most important of all is a careful understanding by the patient of the discipline and demands to be made on her. Success depends largely on the obstetrician's ability to convince her of the necessity of such discipline in her infant's interest.

\section{References}

Pedersen, J. (1952) Diabetes and pregnancy. Blood sugar of newborn infants. M.D. thesis. Danish Science Press, Copenhagen.

Peel, J. (1972) A historical review of diabetes and pregnancy. Journal of Obstetrics and Gynaecology of the British Commonwealth, 79, 385.

Peel, J. \& OAKLey, W.G. (1950) Management of pregnancy in diabetics. Transactions of the 12th Congress of Obstetrics and Gynaecology, 12, 161.

WhITE, P. (1949) Pregnancy complicating diabetes. American Journal of Medicine, 7, 609.

Wright, A.D., DiXon, H.G. \& Joplin, G.F. (1968) Diabetes and latent diabetes in pregnancy. British Medical Bulletin, 24 (i), 25. 